Pacific Journal of Mathematics

EIGENVALUE ESTIMATES WITH APPLICATIONS TO 


\title{
EIGENVALUE ESTIMATES WITH APPLICATIONS TO MINIMAL SURFACES
}

\author{
JOHAN TYSK
}

We study eigenvalue estimates of branched Riemannian coverings of compact manifolds. We prove that if

$$
\varphi: M^{n} \rightarrow N^{n}
$$

is a branched Riemannian covering, and $\left\{\mu_{i}\right\}_{l=0}^{\infty}$ and $\left\{\lambda_{t}\right\}_{i=0}^{\infty}$ are the eigenvalues of the Laplace-Beltrami operator on $M$ and $N$, respectively, then

$$
\sum_{i=0}^{\infty} e^{-\mu_{i} t} \leq k \sum_{i=0}^{\infty} e^{-\lambda_{i} t},
$$

for all positive $t$, where $k$ is the number of sheets of the covering. As one application of this estimate we show that the index of a minimal oriented surface in $\mathbf{R}^{3}$ is bounded by a constant multiple of the total curvature. Another consequence of our estimate is that the index of a closed oriented minimal surface in a flat three-dimensional torus is bounded by a constant multiple of the degree of the Gauss map.

1. Introduction. Motivated by problems in the theory of minimal surfaces, we study the following question. Let

$$
\varphi: M^{n} \rightarrow N^{n}
$$

be a branched Riemannian covering of compact manifolds, which has a singular set of codimension at least two. By this we mean that we endow $M^{n}$ with the pullback metric

$$
\varphi^{*}\left(d s_{N}\right),
$$

where $\varphi^{*}$ is singular on a set of codimension at least two. We then want to estimate the eigenvalues of the Laplace-Beltrami operator on $M$ in terms of the corresponding eigenvalues of $N$. Note that we can speak of the eigenvalues of $\left(M, \varphi^{*}\left(d s_{N}\right)\right)$ although the metric is possibly singular, since a singular set of codimension at least two will not affect the integrals of the variational characterization of the eigenvalues.

Our main theorem gives the estimate

$$
\sum_{i=0}^{\infty} e^{-\mu_{i} t} \leq k \sum_{i=0}^{\infty} e^{-\lambda_{i} t}
$$


for all $t>0$, where $k$ is the number of sheets of the covering $\varphi$, and $\left\{\mu_{i}\right\}$ and $\left\{\lambda_{i}\right\}$ are the eigenvalues of the Laplace-Beltrami operator on $M$ and $N$, respectively. We then use this estimate to show that if $M^{2} \subseteq \mathbf{R}^{3}$ is an oriented complete minimal surface of finite total curvature, then the index of $M$ is bounded by a constant multiple of the total curvature. Here, the index of $M$ is defined to be the limit of the indices of an increasing sequence of exhausting compact domains in $M$. The index of a domain $D$ is the number of negative eigenvalues of the eigenvalue problem

$$
\left(\Delta+|A|^{2}\right) \varphi+\lambda \varphi=0 \text { on } D,\left.\quad \varphi\right|_{\partial D}=0,
$$

where $A$ is the second fundamental form of $M$ as a submanifold of $\mathbf{R}^{3}$. Geometrically, the index of $M$ can be described as the maximum dimension of a linear space of compactly supported deformations that decrease the area up to second order. Finally we also show that the index of a closed oriented minimal surface in a flat three-dimensional torus is bounded by a constant multiple of the degree of the Gauss map.

2. The eigenvalue estimate. Our main result is the following theorem.

THEOREM. Let

$$
\varphi: M^{n} \rightarrow N^{n}
$$

be a $k$-sheeted branched Riemannian covering of compact manifolds, which has a singular set of codimensioin at least two. Let $\left\{\mu_{i}\right\}_{i=0}^{\infty}$ and $\left\{\lambda_{l}\right\}_{i=0}^{\infty}$ be the eigenvalues of the Laplace-Beltrami operator on $M^{n}$ and $N^{n}$, respectively. Then for all $t>0$,

$$
\sum_{i=0}^{\infty} e^{-\mu_{i} t} \leq k \sum_{i=0}^{\infty} e^{-\lambda_{i} t} .
$$

REMARK. Before proving the theorem we note that the main difficulty is that the fundamental comparison theorems of Cheng [1] do not carry through if the metric has singularities. We instead utilize the heat kernel on $M$ and $N$ to circumvent this difficulty.

Proof. We restrict $\varphi$ of the theorem to $\varphi_{-}$:

$$
\varphi_{-}: M_{-} \rightarrow N_{-} \text {, }
$$

where

$$
M_{-}=M-E(\varepsilon)
$$


and $E(\varepsilon)$ is an open set of volume less than $\varepsilon$ with smooth boundary, containing the singular set. We then simply define $N_{-}$to be the image under $\varphi$ restricted to $M_{-}$.

Now fixing $x \in M_{-}$, we consider

$$
H: y \mapsto H_{N_{-}}(\varphi(x), \varphi(y), t), \quad y \in M_{-}, t>0,
$$

where $H_{N_{-}}$is the heat kernel on $N_{-}$, with Dirichlet boundary conditions. Since $\varphi_{-}$is the local isometry, the function $H$ solves the heat equation on $M_{-}$. As $t$ tends to zero we obtain

$$
H_{N_{-}}(\varphi(x), \varphi(y), t) \rightarrow \sum_{\varphi\left(x_{i}\right)=\varphi(x)} \delta_{x_{i}} .
$$

On the other hand, for the heat kernel $H_{M_{-}}$on $M_{-}$with Dirichlet boundary conditions, we have as $t$ tends to zero

$$
H_{M_{-}}(x, y, t) \rightarrow \delta_{x} .
$$

Hence, at $t=0$ we have in the sense of distributions

$$
H_{M_{-}}(x, y, 0) \leq H_{N_{-}}(\varphi(x), \varphi(y), 0) .
$$

By the maximum principle for the heat equation, we then have

$$
H_{M_{-}}(x, y, t) \leq H_{N_{-}}(\varphi(x), \varphi(y), t),
$$

for all $t>0$. Inequality (1) holds for all $x$ and $y$ in $M_{-}$so we can let $x=y$ and integrate over $M_{-}$:

$$
\int_{M_{-}} H_{M_{-}}(x, x, t) d V(x) \leq \int_{M_{-}} H_{N_{-}}(\varphi(x), \varphi(x), t) d V(x) .
$$

Since $\varphi_{-}$is a $k$-sheeted covering, we have

$$
\int_{M_{-}} H_{N_{-}}(\varphi(x), \varphi(x), t) d V(x)=k \int_{N_{-}} H_{N_{-}}(z, z, t) d V(z) .
$$

Again using the maximum principle for the heat equation, we obtain

$$
\int_{N_{-}} H_{N_{-}}(z, z, t) d V(z) \leq \int_{N} H_{N}(z, z, t) d V(z),
$$

where $H_{N}$ denotes the heat kernel of $N$. We have therefore shown that

$$
\int_{M_{-}} H_{M_{-}}(x, x, t) d V(x) \leq k \int_{N} H_{N}(z, z, t) d V(z) .
$$

Finally, letting the volume $\varepsilon$ of $E(\varepsilon)$ tend to zero, we obtain

$$
\int_{M} H_{M}(x, x, t) d V(x) \leq k \int_{N} H_{N}(z, z, t) d V(z),
$$


where $H_{M}$ is the heat kernel of $M$. Using separation of variables, one shows that the heat kernels $H_{M}$ and $H_{N}$ have the representations

$$
\begin{aligned}
& H_{M}(x, y, t)=\sum_{i=0}^{\infty} e^{-\mu_{i} t} \psi_{i}(x) \psi_{i}(y) \\
& H_{N}(x, y, t)=\sum_{i=0}^{\infty} e^{-\lambda_{i} t} \varphi_{i}(x) \varphi_{i}(y),
\end{aligned}
$$

where

$$
\Delta \psi_{i}+\mu_{i} \psi_{i}=0, \quad i=0,1,2, \ldots,
$$

and

$$
\Delta \varphi_{i}+\lambda_{i} \varphi_{i}=0, \quad i=0,1,2, \ldots,
$$

are the eigenvalues and eigenfunctions of $M$ and $N$, respectively, normalized so that $\left\{\psi_{i}\right\}_{i=0}^{\infty}$ and $\left\{\varphi_{i}\right\}_{i=0}^{\infty}$ form orthonormal systems. Using these representations in inequality $(2)$, we obtain

$$
\sum_{i=0}^{\infty} e^{-\mu_{i} t} \leq k \sum_{i=0}^{\infty} e^{-\lambda_{t} t}
$$

finishing the proof of the theorem.

3. Applications to minimal surfaces. In [2], D. Fisher-Colbrie shows that a complete minimal oriented surface $M$ in $\mathbf{R}^{3}$ has finite index if and only if it has finite total curvature (see the introduction for the definition of index). A natural question to ask then is how the index varies with the total curvature. Using our eigenvalue estimate, we can show that the index is bounded by a constant multiple of the total curvature.

THEOREM. Let $M^{2}$ be a complete oriented minimal surface in $\mathbf{R}^{3}$. Set

$$
k=\frac{1}{4 \pi} \int_{M}(-K) d V,
$$

where $K$ is the Gaussian curvature of $M$. Then

$$
\text { index of } M \leq(7.68183) \cdot k \text {. }
$$

Proof. Without loss of generality, we can assume that $k$ is finite. By Osserman's classical theorem, we then know that $M$ is conformally a compact Riemann surface $\bar{M}$, punctured at a finite set of points. Also, the Gauss map extends to a conformal map

$$
G: \bar{M} \rightarrow S^{2} .
$$


For a minimal surface in $\mathbf{R}^{3},|A|^{2}=-2 K$. Now, the number of negative eigenvalues for

$$
\Delta+|A|^{2}=\Delta-2 K,
$$

on any domain $D$ in $M$, is the same as the number of negative eigenvalues of the corresponding domain in $\bar{M}$ for the operator

$$
\Delta_{\bar{M}}+2 \text {, }
$$

where we use the pullback metric from $S^{2}$ on $\bar{M}$. This follows from the fact that

$$
G^{*}\left(d s_{S^{2}}^{2}\right)=(-K) \cdot d s_{M}^{2},
$$

and $\Delta_{M}=(-K) \Delta_{\bar{M}}$. Since the index of $M$ is the limit of the indices of an exhausting sequence of domains $D$ in $M$, we can conclude, by the domain monotonicity of eigenvalues, that the index of $M$ is bounded by the number of negative eigenvalues of $\Delta_{\bar{M}}+2$ on $\bar{M}$, or equivalently, by the number of eigenvalues of $\Delta_{\bar{M}}$ that are strictly less than two.

Now, $G$ is a holomorphic mapping so it establishes $\bar{M}$ as a $k$-sheeted branched cover of $S^{2}$. The singular set of this covering is the set of isolated points where $K=0$. We can therefore apply our eigenvalue estimate and conclude that

$$
\sum_{i=0}^{\infty} e^{-\mu_{i} t} \leq k \sum_{i=0}^{\infty} e^{-\lambda_{i} t}, \quad \text { all } t>0,
$$

where $\left\{\mu_{i}\right\}_{i=0}^{\infty}$ and $\left\{\lambda_{i}\right\}_{i=0}^{\infty}$ are the eigenvalues of $\bar{M}$ and $S^{2}$, respectively. Since the index of $M$ is bounded by the number of $\mu_{i}$ 's that are strictly less than two, we conclude that

$$
(\text { index of } M) \cdot e^{-2 t} \leq \sum_{\mu_{i}<2} e^{-\mu_{i} t} \leq \sum_{i=0}^{\infty} e^{-\mu_{i} t} \leq k \sum_{i=0}^{\infty} e^{-\lambda_{i} t} .
$$

Hence

$$
\text { index of } M \leq\left(e^{2 t} \sum_{i=0}^{\infty} e^{-\lambda_{\imath} t}\right) \cdot k \text {. }
$$

The $i$ th distinct eigenvalue of $S^{2}$ is known to be $i(i+1)$, with multiplicity $2 i+1$. Using this, we find that $t=0.4506 \ldots$ gives the smallest possible value of $7.68182 \ldots$ for the coefficient of $k$, proving the theorem.

As another application of our eigenvalue estimate, we consider the case of minimal surfaces in a flat three-dimensional torus. Let $N$ be such a torus, which we know we can write isometrically as

$$
N=\mathbf{R}^{3} / \Lambda
$$


where $\Lambda$ is a cocompact lattice, and let $M$ be a closed minimal oriented surface immersed in $N$. We can define the Gauss map

$$
G: M \rightarrow S^{2}
$$

by viewing $M$ as a minimal surface in $\mathbf{R}^{3}$, periodic with respect to the lattice $\Lambda$.

The index of $M$ is the number of negative eigenvalues of

$$
\Delta+|A|^{2}=\Delta-2 K
$$

on $M$, where $A$ denotes the second fundamental form of $M$ in $N$, and $K$ denotes the Gaussian curvature of $M$. We endow $M$ with the pullback metric from $S^{2}$ via $G$ and conclude, using the same argument as in the preceding example, that

$$
\text { index of } M \leq(7.68183) \cdot k \text {, }
$$

where $k$ is the degree of the Gauss map.

\section{REFERENCES}

[1] S. Y. Cheng, Eigenvalue comparison theorems and its geometric applications, Math. Z., 143 (1975), 289-297.

[2] D. Fischer-Colbrie, On complete minimal surfaces with finite index in three manifolds, preprint.

Received July 14, 1986.

UNIVERSITY OF CALIFORNIA, LOS ANGELES

LOS ANGELES, CA 90024 


\title{
PACIFIC JOURNAL OF MATHEMATICS EDITORS
}

\author{
V. S. VARADARAJAN \\ (Managing Editor) \\ University of California \\ Los Angeles, CA 90024 \\ HERBERT Clemens \\ University of Utah \\ Salt Lake City, UT 84112 \\ R. FINN \\ Stanford University \\ Stanford, CA 94305
}

\author{
HERMANN FLASCHKA \\ University of Arizona \\ Tucson, AZ 85721 \\ RAMESH A. GANGOLLI \\ University of Washington \\ Seattle, WA 98195 \\ VAUGHAN F. R. JONES \\ University of California \\ Berkeley, CA 94720 \\ ROBION KIRBY \\ University of California \\ Berkeley, CA 94720
}

\author{
C. C. Moore \\ University of California \\ Berkeley, CA 94720
}

H. SAMELSON

Stanford University

Stanford, CA 94305

HAROLD STARK

University of California, San Diego

La Jolla, CA 92093

\section{ASSOCIATE EDITORS}

\author{
R. ARENS \\ E. F. BECKENBACH \\ B. H. NEUMANN \\ F. WOLF \\ K. YOSHIDA \\ (1906-1982)

\section{SUPPORTING INSTITUTIONS} \\ UNIVERSITY OF ARIZONA \\ UNIVERSITY OF BRITISH COLUMBIA \\ CALIFORNIA INSTITUTE OF TECHNOLOGY \\ UNIVERSITY OF CALIFORNIA \\ MONTANA STATE UNIVERSITY \\ UNIVERSITY OF NEVADA, RENO \\ NEW MEXICO STATE UNIVERSITY \\ OREGON STATE UNIVERSITY

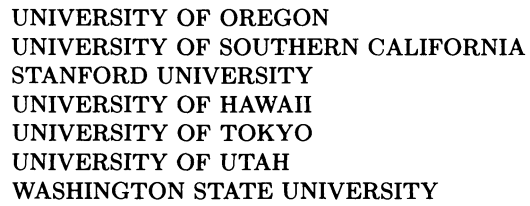

The Supporting Institutions listed above contribute to the cost of publication of this Journal, but they are not owners or publishers and have no responsibility for its content or policies.

Mathematical papers intended for publication in the Pacific Journal of Mathematics should be in typed form or offset-reproduced (not dittoed), double spaced with large margins. Please do not use built up fractions in the text of the manuscript. However, you may use them in the displayed equations. Underline Greek letters in red, German in green, and script in blue. The first paragraph must be capable of being used separately as a synopsis of the entire paper. In particular it should contain no bibliographic references. Please propose a heading for the odd numbered pages of less than 35 characters. Manuscripts, in triplicate, may be sent to any one of the editors. Please classify according to the scheme of Math. Reviews, Index to Vol. 39. Supply name and address of author to whom proofs should be sent. All other communications should be addressed to the managing editor, or Elaine Barth, University of California, Los Angeles, California 90024.

There are page-charges associated with articles appearing in the Pacific Journal of Mathematics. These charges are expected to be paid by the author's University, Government Agency or Company. If the author or authors do not have access to such Institutional support these charges are waived. Single authors will receive 50 free reprints; joint authors will receive a total of $\mathbf{1 0 0}$ free reprints. Additional copies may be obtained at cost in multiples of 50 .

The Pacific Journal of Mathematics is issued monthly as of January 1966. Regular subscription rate: $\$ 190.00$ a year (5 Vols., 10 issues). Special rate: $\$ 95.00$ a year to individual members of supporting institutions.

Subscriptions, orders for numbers issued in the last three calendar years, and changes of address should be sent to Pacific Journal of Mathematics, P.O. Box 969, Carmel Valley, CA 93924, U.S.A. Old back numbers obtainable from Kraus Periodicals Co., Route 100, Millwood, NY 10546.

The Pacific Journal of Mathematics at P.O. Box 969, Carmel Valley, CA 93924 (ISSN 0030-8730) publishes 5 volumes per year. Application to mail at Second-class postage rates is pending at Carmel Valley, California, and additional mailing offices. Postmaster: send address changes to Pacific Journal of Mathematics, P.O. Box 969, Carmel Valley, CA 93924.

PUBLISHED BY PACIFIC JOURNAL OF MATHEMATICS, A NON-PROFIT CORPORATION Copyright (C) 1987 by Pacific Journal of Mathematics 


\section{Pacific Journal of Mathematics}

Vol. 128 , No. $2 \quad$ April, 1987

Pierre Barrucand, John Harold Loxton and Hugh C. Williams, Some

explicit upper bounds on the class number and regulator of a cubic field

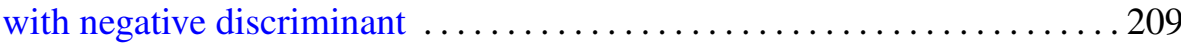

Thomas Ashland Chapman, Piecewise linear fibrations $\ldots \ldots \ldots \ldots \ldots 223$

Yves Félix and Jean-Claude Thomas, Extended Adams-Hilton's

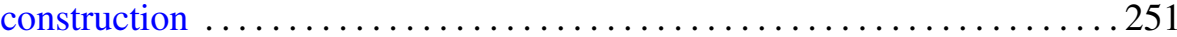

Robert Fitzgerald, Derivation algebras of finitely generated Witt rings . . . 265

K. Gopalsamy, Oscillatory properties of systems of first order linear delay differential inequalities ................................ 299

John P. Holmes, One parameter subsemigroups in locally complete

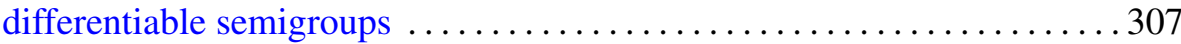

Douglas Murray Pickrell, Decomposition of regular representations for

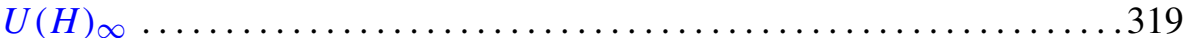

Victoria Powers, Characterizing reduced Witt rings of higher level

Parameswaran Sankaran and Peter Zvengrowski, Stable parallelizability

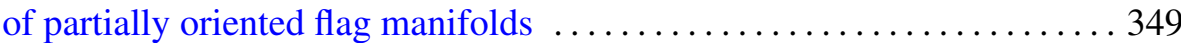

Johan Tysk, Eigenvalue estimates with applications to minimal surfaces . . . 361 Akihito Uchiyama, On McConnell's inequality for functionals of subharmonic functions

Minato Yasuo, Bott maps and the complex projective plane: a construction

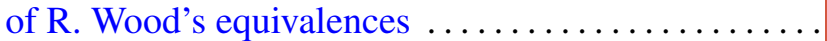

James Juei-Chin Yeh, Uniqueness of strong solutions to stochastic differential equations in the plane with deterministic boundary process 